Component-wise deuterium-tritium fusion yield predictions with neutron emission spectrometry

Sahlberg, A.

2019

Sahlberg , A , Eriksson , J , Conroy , S , Ericsson , G , Hellesen , C \& King , D 2019 , '

Component-wise deuterium-tritium fusion yield predictions with neutron emission

spectrometry ' , Nuclear Fusion , vol. 59 , no. 12 . https://doi.org/10.1088/1741-4326/ab45a6

http://hdl.handle.net/10138/324317

https://doi.org/10.1088/1741-4326/ab45a6

acceptedVersion

Downloaded from Helda, University of Helsinki institutional repository.

This is an electronic reprint of the original article.

This reprint may differ from the original in pagination and typographic detail.

Please cite the original version. 


\title{
Component-wise DT fusion yield predictions with neutron emission spectrometry
}

\author{
A. Sahlberg ${ }^{1}$, J. Eriksson ${ }^{1}$, S. Conroy ${ }^{1}$, G. Ericsson ${ }^{1}$, \\ C. Hellesen ${ }^{1}$, D. King ${ }^{2}$ and JET Contributors* \\ ${ }^{1}$ Dept. of Physics and astronomy, Uppsala University, Sweden \\ ${ }^{2}$ CCFE, Culham Science Centre, Abingdon, Oxfordshire, UK
}

\begin{abstract}
This paper uses neutron emission spectrometry (NES), with the spectrometer TOFOR, to estimate the deuteriumtritium (DT) equivalent fusion yields of deuterium-only (DD) pulses at the tokamak JET. A method for making DT predictions using parameters determined from the neutron energy spectrum is described and this method is compared to corresponding estimations done with the modeling codes JESTORR and TRANSP, as well as with results from the 1997 JET DT campaign. The method has been applied to a large number of JET DD pulses conducted after the installation of the ITER-like wall (ILW), and the results have been used to assess the prospects for the upcoming DT campaign (DTE2).

DT predictions made by NES produces similar estimations as JESTORR and TRANSP, and the fusion power from studied DT pulses fall in line with the estimated power of similar DD discharges.

Extrapolating the recent JET pulses to estimate what the fusion yields would be in DTE2, results indicate that reaching over $10 \mathrm{MW}$ is achievable and that the highest performing ILW pulses to date could approach the desired fusion power of $15 \mathrm{MW}$.
\end{abstract}

\section{Introduction}

Since JET's previous DT campaign DTE1, over twenty years ago, there have been no high-performing deuterium-tritium (DT) pulses in the world's largest tokamak. Now, in preparation for ITER, there is a second DT campaign scheduled, DTE2[1]. This campaign plans to have more heating power and reach a fusion power of $15 \mathrm{MW}$, sustained for a few seconds, as opposed to DTE1[2] where this power was reached for just an instant.

To prepare for this new DT campaign, we seek the ability to make quick and robust predictions of DT fusion yields. There are many approaches to DT extrapolation, i.e., analyzing a deuterium-only (DD) pulse and estimating what fusion yields a comparable shot would have resulted in if it was operated with DT fuel instead. One relevant concept is that of DT equivalence[3], which was introduced in the 1990s, when making simulations for DT predictions on the tokamak TFTR. It can be estimated in a number of ways, commonly one uses a plasma modeling code to simulate the fusion reaction rate of a given DD plasma discharge twice, once with only deuterium, and once with half the fuel replaced by tritium. Then the DT equivalent fusion power is obtained as

$$
P_{\mathrm{DTeq}}=R_{\text {measured }} \frac{R_{\text {modeled,DT }}}{R_{\text {modeled,DD }}} \times 17.6 \mathrm{MeV}
$$

where $R$ represents reaction rate, $P$ is the fusion power and $17.6 \mathrm{MeV}$ is the Q-value for DT converting from reaction rate to fusion power.

When calculating DT equivalent fusion power, one assumes the pulse is identical to the D discharge, but with a 50/50 deuterium and tritium mixture in both the bulk plasma and in the population of neutral beam injected (NBI) ions. To make the estimate more conservative, and the modeling easier, one does not take $\alpha$-particle heating or similar such effects into account.

The aim of this paper is to develop a method that uses neutron emission spectrometry (NES) for DT predictions and to apply the said method to recent high-performing JET pulses to aid the preparations for the upcoming DT campaign. The time-of-flight neutron spectrometer TOFOR[4] is used to measure

\footnotetext{
* See the author list of E. Joffrin et al. accepted for publication in Nuclear Fusion Special issue 2019, https://doi.org/10.1088/1741-4326/ab2276
} 
the neutron spectrum. By analyzing the energy spectrum of emitted neutrons, we identify intensities of the different reaction components (thermonuclear, beam-target and RF-target), and use information obtained from this analysis to determine how the component reaction rates scale when going from a DD to a DT plasma.

A large number of JET pulses have been analyzed, and the estimated DT equivalent fusion yields have been compared to other DT extrapolations done using the codes JESTORR[5] and TRANSP[6]. A comparison with actual JET DT discharges from DTE1 has also been performed.

\section{Neutron emission rates from D and DT plasmas}

The aim is to estimate the expected increase in reaction rate when changing from a deuterium-only pulse to one with both deuterium and tritium. The reaction rate in a fusion plasma can be calculated as

$$
R=\frac{n_{a} n_{b}}{1+\delta_{a, b}}\langle\sigma v\rangle
$$

where $n_{i}$ is the ion density of ion $i$ and $\delta_{a, b}$, the Kroenecker delta function, is used to avoid double counting particles when they are of the same population. The $\langle\sigma v\rangle$ is the reactivity between particle $a$ and $b$, calculated by averaging the product of the cross section and the relative velocity, i.e.

$$
\langle\sigma v\rangle=\iint_{\mathbb{R}^{3}}\left\|\boldsymbol{v}_{a}-\boldsymbol{v}_{b}\right\| \sigma\left(\boldsymbol{v}_{a}, \boldsymbol{v}_{b}\right) f_{a}\left(\boldsymbol{v}_{a}\right) f_{b}\left(\boldsymbol{v}_{b}\right) \mathrm{d} \boldsymbol{v}_{a} \mathrm{~d} \boldsymbol{v}_{b}
$$

where $f_{i}$ is the velocity distribution function of ion species $i$. In this expression both $f_{a}$ and $f_{b}$ are normalized to one, since we multiply this expression by the densities in (2), so only the shape of the ion distributions are needed to calculate the reactivity.

Each of the reaction components has their reaction rate, and for each component, a scale-up factor for going from a D to a DT plasma can be defined as

$$
S=\frac{R_{\mathrm{DT}}}{R_{\mathrm{DD}}},
$$

i.e. the fraction between the DT and DD reaction rates from equation (1). This factor depends on the velocity distribution of the reacting ions, and a fusion plasma generally has sub-populations of ions with different velocity distributions. In this derivation we will assume a general case where two sub-populations $a$ and $b$ interact. Two cases are considered, one where both populations consist entirely of D and one where each population contains both $\mathrm{D}$ and $\mathrm{T}$. The corresponding reaction rates are

$$
\begin{array}{r}
R_{\mathrm{DD}}=\frac{n_{a} n_{b}}{1+\delta_{a, b}}\langle\sigma v\rangle_{a b, \mathrm{DD}} \\
R_{\mathrm{DT}}=\frac{n_{a, \mathrm{D}} n_{b, \mathrm{~T}}}{1+\delta_{a, b}}\langle\sigma v\rangle_{a b, \mathrm{DT}}+\frac{n_{a, \mathrm{~T}} n_{b, \mathrm{D}}}{1+\delta_{a, b}}\langle\sigma v\rangle_{a b, \mathrm{TD}}
\end{array}
$$

for the DD and DT reactions respectively. Note that to find the total DT reaction rate here we must add the rates of reactions with deuterium from $a$ and tritium from $b$ with the one with tritium from $a$ and deuterium from $b$. Hence the reaction rate scale up factor is

$$
S_{\mathrm{AB}}=\frac{R_{\mathrm{DT}}}{R_{\mathrm{DD}}}=\frac{n_{a, \mathrm{D}} n_{b, \mathrm{~T}}\langle\sigma v\rangle_{a b, \mathrm{DT}}+n_{a, \mathrm{~T}} n_{b, \mathrm{D}}\langle\sigma v\rangle_{a b, \mathrm{TD}}}{n_{a} n_{b}\langle\sigma v\rangle_{a b, \mathrm{DD}}} .
$$

Now let us assume that both sub-populations have a the same total density in both the DD and DT case, i.e.

$$
\begin{array}{r}
n_{a}=n_{a, \mathrm{D}}+n_{a, \mathrm{~T}} \\
n_{b}=n_{b, \mathrm{D}}+n_{b, \mathrm{~T}}
\end{array}
$$

If the fuel ion ratio in the DT plasma is $\frac{n_{\mathrm{i}, \mathrm{T}}}{n_{\mathrm{i}, \mathrm{D}}}=k_{\mathrm{i}}$ for the populations $i \in\{a, b\}$, we can cancel out the densities in equation (7) and rewrite it as

$$
S_{\mathrm{AB}}=\frac{k_{b}\langle\sigma v\rangle_{a b, \mathrm{DT}}+k_{a}\langle\sigma v\rangle_{a b, \mathrm{TD}}}{\left(1+k_{\mathrm{a}}\right)\left(1+k_{\mathrm{b}}\right)\langle\sigma v\rangle_{a b, \mathrm{DD}}}
$$


and hence get an expression independent of the fuel ion densities.

We now have a general expression for the scale-up factor for the reaction component from two arbitrary fuel ion distributions. To calculate it we need the shape of the velocity distributions $a, b$ as well as the fuel ion ratio of the populations. Note that we do not need the density of the populations since the distributions are normalized to one in (3), and therefore knowing the shapes of the distributions is enough.

The most relevant fuel ion populations in a fusion plasma are thermal ions (also called bulk ions), and high-energy ions injected by neutral beam injection (NBI)[7], or accelerated by ion-cyclotron radiofrequency (RF) heating[8]. Fusion reactions between ions from these distributions will all contribute to the neutron emission, and the three most significant reaction components in a high-performance JET fusion plasma are thermonuclear reactions, beam-target (BT) reactions, and RF-target reactions (i.e. RFaccelerated ions that react with the bulk plasma). These components will scale up differently when going from a D to a DT plasma. There are likewise beam-beam, RF-RF and beam-RF reaction components, but these are generally negligible for high-density plasmas, such as the high performance pulses that are the focus of this paper, and are ignored in this analysis.

For the thermonuclear component, we have ions with a Maxwellian distribution interacting with other ions from the same distribution. One assumption usually made when estimating DT equivalent fusion power is that there is equal amounts of deuterium and tritium in the fuel, i.e., a fuel ion ratio of one. Under these assumptions (10) collapses into

$$
S_{\mathrm{th}}=\frac{\langle\sigma v\rangle_{\mathrm{th}, \mathrm{DT}}}{2\langle\sigma v\rangle_{\mathrm{th}, \mathrm{DD}}} .
$$

Since the reactivities are obtained here by averaging over Maxwellian distributions, this expression only depends on the plasma temperature, which can be determined from the neutron emission spectrum, or some other diagnostic.

The beam-target component's scale up factor becomes, upon assuming a fuel ion ratio of one in both the bulk plasma and the NBI slowing down population,

$$
S_{\mathrm{bt}}=\frac{\langle\sigma v\rangle_{\mathrm{bt}, \mathrm{DT}}+\langle\sigma v\rangle_{\mathrm{bt}, \mathrm{TD}}}{4\langle\sigma v\rangle_{\mathrm{bt}, \mathrm{DD}}},
$$

where $\langle\sigma v\rangle_{\mathrm{bt}, \mathrm{DT}}$ and $\langle\sigma v\rangle_{\mathrm{bt}, \mathrm{TD}}$ are the beam-target reactivities for beam deuterium reacting with bulk tritium and vice versa, calculated from (3). The expression in (12) depends both on the plasma temperature and the shape of the slowing down distribution of the beam ions, which needs to be modeled. Often TRANSP/NUBEAM[9] is used to determine the beam slowing down distribution, however running such an extensive simulation is not needed for this method, since it only requires the shape of the said distribution, and in this paper it has been has obtained it by solving a one-dimensional Fokker-Planck equation[10]. Note that this equation is solved separately for beam-injected deuterons and tritons to account for their different slowing-down distributions.

The RF-target reaction comes from RF-accelerated fast ions reacting with the thermal bulk-ions. The situation is similar to that of the beam-target reaction, i.e., we have two ion populations interacting with each other. However, due to the way RF heating works by resonating with the ions cyclotron frequency[8], it will heat either deuterium or tritium ions, and hence the fuel ion ratio $\frac{n_{\mathrm{T}}}{n_{\mathrm{D}}}$ in the population of RF-ions will in reality either be close to infinity or zero, depending on which ion is accelerated. The RF-target scale-up factor follows from (10) and is expressed as

$$
S_{\mathrm{rf}}= \begin{cases}\frac{\langle\sigma v\rangle_{\mathrm{rf}, \mathrm{DT}}}{2\langle\sigma v\rangle_{\mathrm{rf}, \mathrm{DD}}}, & \text { if D-ions are accelerated } \\ \frac{\langle\sigma v v\rangle_{\mathrm{rf}} \mathrm{TD}}{2\langle\sigma v\rangle_{\mathrm{rf}, \mathrm{DD}}}, & \text { if T-ions are accelerated. }\end{cases}
$$

In the analysis performed in this paper, we have assumed deuterium ions to be accelerated by RF heating. To calculate these expressions, the shapes of the velocity distributions of both ion species are needed. In this paper, an anisotropic Maxwellian energy distribution with pitch-angles between 80-100 degrees is used for the superheated RF-ions with a temperature determined from neutron spectrometry.

So the final expressions for all the component scale-up factors are equations (11), (12), and (13). To calculate them one needs the shapes of the ion velocity distributions, and the energy-dependent cross sections for the DD and DT reactions. The scale-up factor for the thermonuclear, beam-target and RF-target reactions, under the above assumptions about fuel ion ratios, are shown in figure 1. 


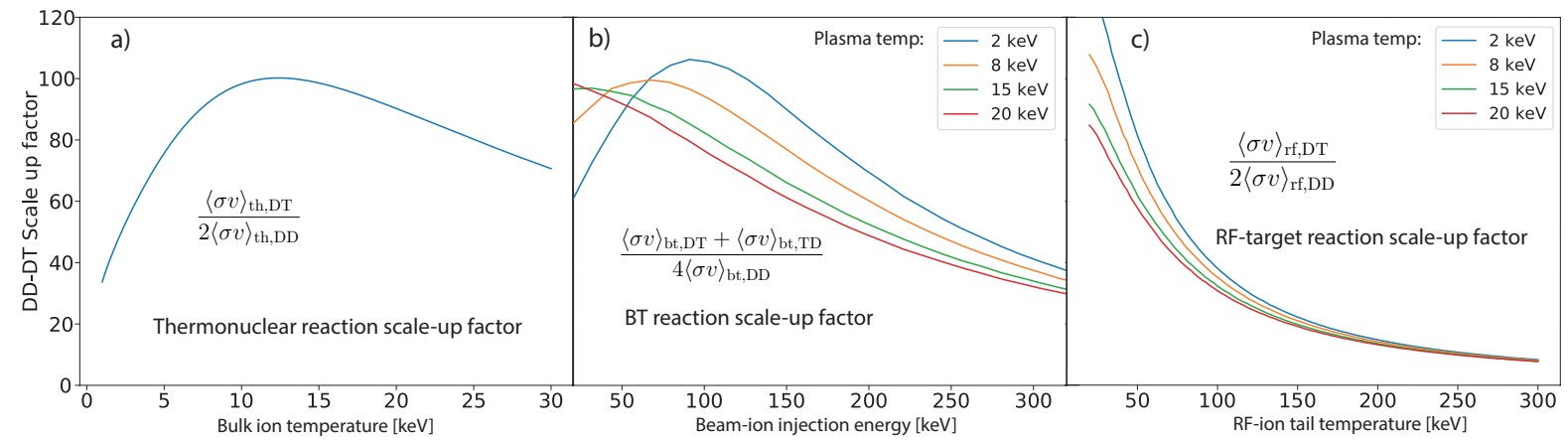

Fig. 1: The DD to DT scale-up factors for the thermonuclear (a), beam-target (b) and RF-target (c) reaction rates. A 50/50 deuterium-tritium plasma is assumed. The beam-target and RF scale-up factors are shown for different plasma temperatures.

With the scale-up factors, one can extrapolate the fusion yield from the different reaction components in a D pulse and get an estimation of the yield in a similar DT pulse. The total scale-up factor is found by weighting the scale-up factors for each component with their respective intensities, according to the expression

$$
S_{\mathrm{tot}}=\frac{S_{\mathrm{th}} I_{\mathrm{th}}+S_{\mathrm{bt}} I_{\mathrm{bt}}+S_{\mathrm{rf}} I_{\mathrm{rf}}}{I_{\mathrm{th}}+I_{\mathrm{bt}}+I_{\mathrm{rf}}}
$$

where $I_{\mathrm{th}}, I_{\mathrm{bt}}$, and $I_{\mathrm{rf}}$ are the reaction intensities of the thermal, beam-target and RF-reactions. The component reaction intensities are in this paper determined from NES.

The expected total DT fusion yield is found by multiplying the measured neutron rate of a DD pulse by the total scale-up factor.

\section{Method}

\subsection{Neutron emission spectrometry}

The neutrons arising from the fusion reactions have an energy, $E_{n}$, that depends on the reactant velocities. In the lab frame it can be expressed as[11]

$$
E_{n}=\frac{m_{r}}{m_{n}+m_{r}}(Q+K)+\frac{1}{2} m_{n} v_{\mathrm{cm}}^{2}+v_{\mathrm{cm}} \cos \theta\left[\frac{2 m_{n} m_{r}}{m_{n}+m_{r}}(Q+K)\right]^{1 / 2}
$$

where $K$ is the relative kinetic energy of the reactants, $m_{n}$ and $m_{r}$ the masses of the fusion produced neutron and the $H e$ nucleus respectively, $v_{\mathrm{cm}}$ the mass centre velocity and $\theta$ the angle between the emitted neutron direction in the centre-of-mass frame and $v_{\mathrm{cm}}$. If we have an estimation of the velocity distributions of the reacting ion populations, we can find the expected energy spectrum of neutrons emitted in a direction, specified by the angle $\theta$ in (15).

For example, if we assume a Maxwellian distribution and derive the statistical moments for the distribution of $E_{n}$, we see that the energy spectrum of neutrons from thermonuclear fusion reactions can be analytically described by the expression[11]

$$
N_{\mathrm{th}}\left(E_{\mathrm{n}}\right)=I_{\mathrm{th}} \exp \left[-\frac{m_{1}+m_{2}}{m_{\mathrm{n}}} \frac{\left(E_{\mathrm{n}}-\left\langle E_{\mathrm{n}}\right\rangle\right)^{2}}{4 k_{\mathrm{b}} T\left\langle E_{\mathrm{n}}\right\rangle}\right],
$$

where $m_{1}$ and $m_{2}$ are reactant masses, $m_{\mathrm{n}}$ is the neutron mass, and $\left\langle E_{\mathrm{n}}\right\rangle$ is the average neutron energy, i.e. 2.5 MeV for DD and $14 \mathrm{MeV}$ for DT. In other words, we can model the thermonuclear spectral component as a normal distribution with two independent parameters: the temperature $T$ and the relative intensity $I_{\mathrm{th}}$.

The neutrons emitted from the BT and RF reactions will have unique signatures in the neutron energy spectra. Just like the thermonuclear component, these spectra can, if we have the ion velocity distributions, be inferred from (15).

In this paper, neutron spectra were measured with the time-of-flight neutron spectrometer TOFOR at JET[4]. TOFOR looks down through the core of the plasma from above the roof of the reactor hall. An 
example of calculated neutron energy spectra from the thermonuclear, BT and RF reactions, as seen from the TOFOR line-of-sight, is presented in figure $2 \mathrm{~b}$. The modeled spectrum of backscattered neutrons (i.e. neutrons scattering off the reactor walls into the detector) is also included[12].

To determine the reaction component intensities, modeled spectral components are fitted to the TOFOR data, an example of which is shown in figure 2a. To this end, the modeled neutron energy spectra are multiplied with the TOFOR response matrix[13], which maps the incoming energy spectra to time-of-flight response.

The parameters that describe the modeled spectra are fitted so that their folded sum matches the TOFOR data. Such a fit is displayed in figure 2a.
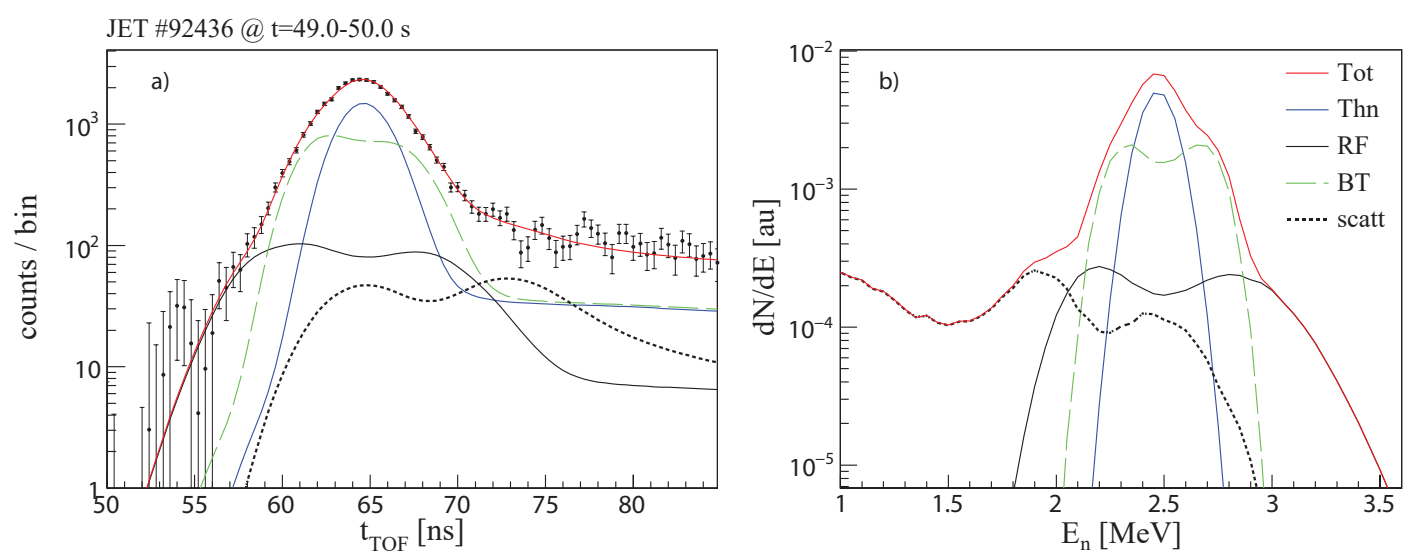

Fig. 2: The neutron time-of-flight spectrum measured by TOFOR for JET discharge 92436 (a) and its corresponding energy spectrum (b). The different spectral components fitted to the measurement are shown.

In this paper neutron emission spectrometry is utilized to determine the relative reaction component intensities, as well as to estimate the bulk plasma temperature and the tail temperature of the RF accelerated deuterons. We use these results to determine the total neutron rate scale-up factor from equation (14) and hence estimate the DT equivalent fusion power.

\subsection{Analysis method}

Let us, as an illustration of the method outlined above, consider JET discharge 92432. The neutron timeof-flight spectrum measured by TOFOR is displayed in figure $3 \mathrm{a}$ and the shot is summarized in table 1. To determine our sought parameters using this spectrum, we must calculate the shape of the energy spectra of the neutron emissions, and this begins with modeling the shape of the underlying fuel-ion distributions, which are shown in figure 3b. These distributions depend on several underlying parameters (c.f. section 2), two of which (the plasma temperature, $T_{i}$ and the RF-ion temperature, $T_{\mathrm{rf}}$ ) we want to determine.

Tab. 1: A summary of JET pulse 92432.

\begin{tabular}{ccccccc} 
Pulse & $B_{T} \mathrm{~T}$ & $I_{p}$ MA & $n_{e} \mathrm{~m}^{-3}$ & $T_{e} \mathrm{keV}$ & $P_{\text {Heat }}$ MW & $R_{\text {neut }} \mathrm{s}^{-1}$ \\
\hline 92432 & 2.8 & 3.0 & $8 \times 10^{19}$ & 6.2 & 32.3 & $2 \times 10^{16}$
\end{tabular}



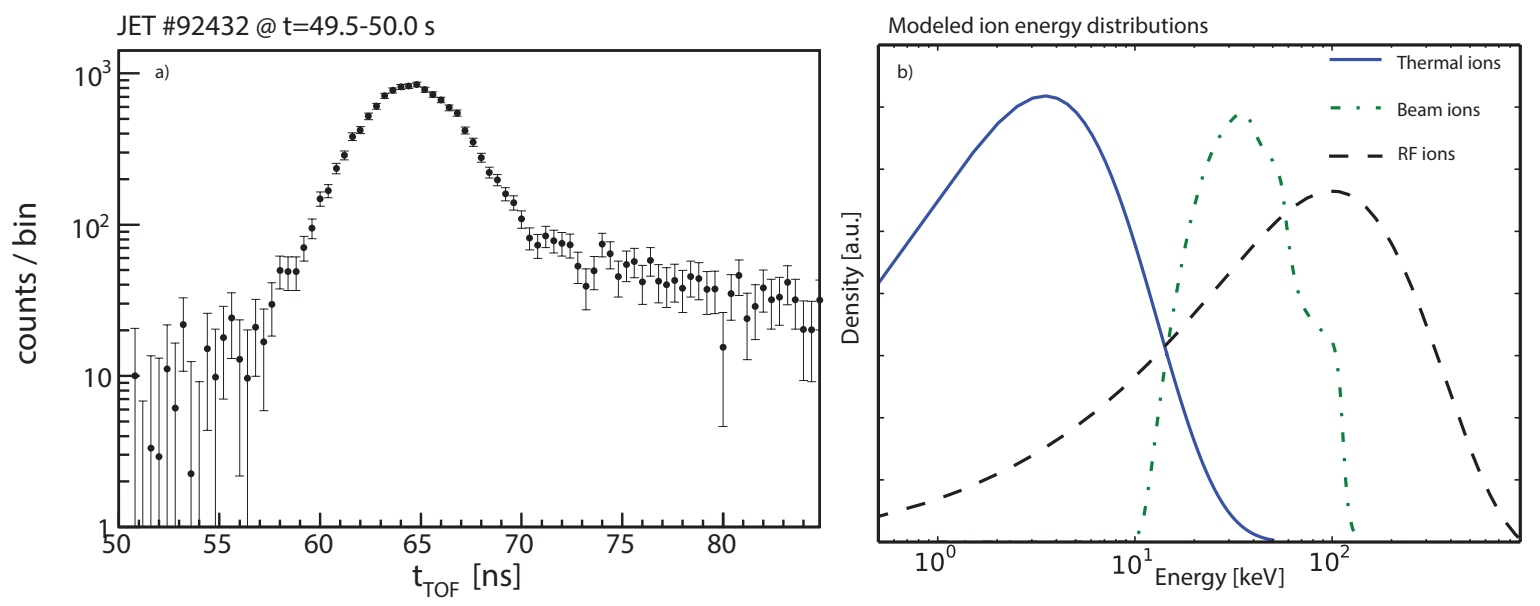

Fig. 3: (a) The neutron time-of-flight spectra for pulse 92432, measured with TOFOR. (b) The modeled shapes of the ion energy distributions for the thermal, the beam and the RF-accelerated ions.

Once we have a model of the ion distribution shapes, these are used to determine the shape of the neutron emission spectra for each reaction component. We calculate the thermonuclear part as equation (16), and use Monte Carlo methods[14][15] to determine the beam-target and RF-target component spectra. The backscattered neutrons are likewise incorporated in the total spectrum, the modeling of which is detailed in [12].

The spectral components are functions of $T_{i}, T_{\mathrm{rf}}, I_{\mathrm{th}}, I_{\mathrm{bt}}$, and $I_{\mathrm{rf}}$, and by multiplying the componentspectra with the TOFOR response matrix and minimizing the Cach-statistic[16], we obtain an assessment of the neutron spectrum components and the fitting parameters. The fit performed on shot 92432 is presented in figure 4 , and the fitted parameters are shown in table 2.

Tab. 2: The values of bulk-ion temperature $\left(T_{i}\right)$, RF-ion temperature $\left(T_{\mathrm{rf}}\right)$, relative intensities of the reaction components, $\left(I_{\mathrm{th}}, I_{\mathrm{bt}}\right.$ and $\left.I_{\mathrm{th}}\right)$ and the fraction of backscattered neutron registering in the detector, obtained by fitting the reaction components to the time-of-flight spectra.

\begin{tabular}{cclllc}
\hline$T_{i}[\mathrm{keV}]$ & $T_{\mathrm{rf}}[\mathrm{keV}]$ & $I_{\mathrm{th}}$ & $I_{\mathrm{bt}}$ & $I_{\mathrm{rf}}$ & Scatt frac \\
\hline $5.0(1)$ & $127(27)$ & $0.39(2)$ & $0.51(3)$ & $0.10(2)$ & $0.10(2)$
\end{tabular}

We enter these parameters into equations (11), (12), (13), and (14) and a scale-up factor for each component, and in turn the one for the total neutron rate, is calculated. The DT equivalent fusion power is found by inserting the scale-up factor into equation (1), i.e., scaling up the neutron rate measured by fission chambers and multiplying by the energy released per reaction.

Table 3.2 shows the obtained scale-up factors. The entire analysis process is displayed in figure 4 .

Tab. 3: The fitted relative intensity and the calculated neutron rate scale-up factors for each reaction component.

\begin{tabular}{l|cc} 
Component & Relative intensity & Scale-up factor \\
\hline Thermonuclear & $0.39(2)$ & $74(4)$ \\
RF-target & $0.10(2)$ & $27(10)$ \\
Beam-Target & $0.51(3)$ & $102(3)$ \\
Tot & 1 & $84(2)$
\end{tabular}



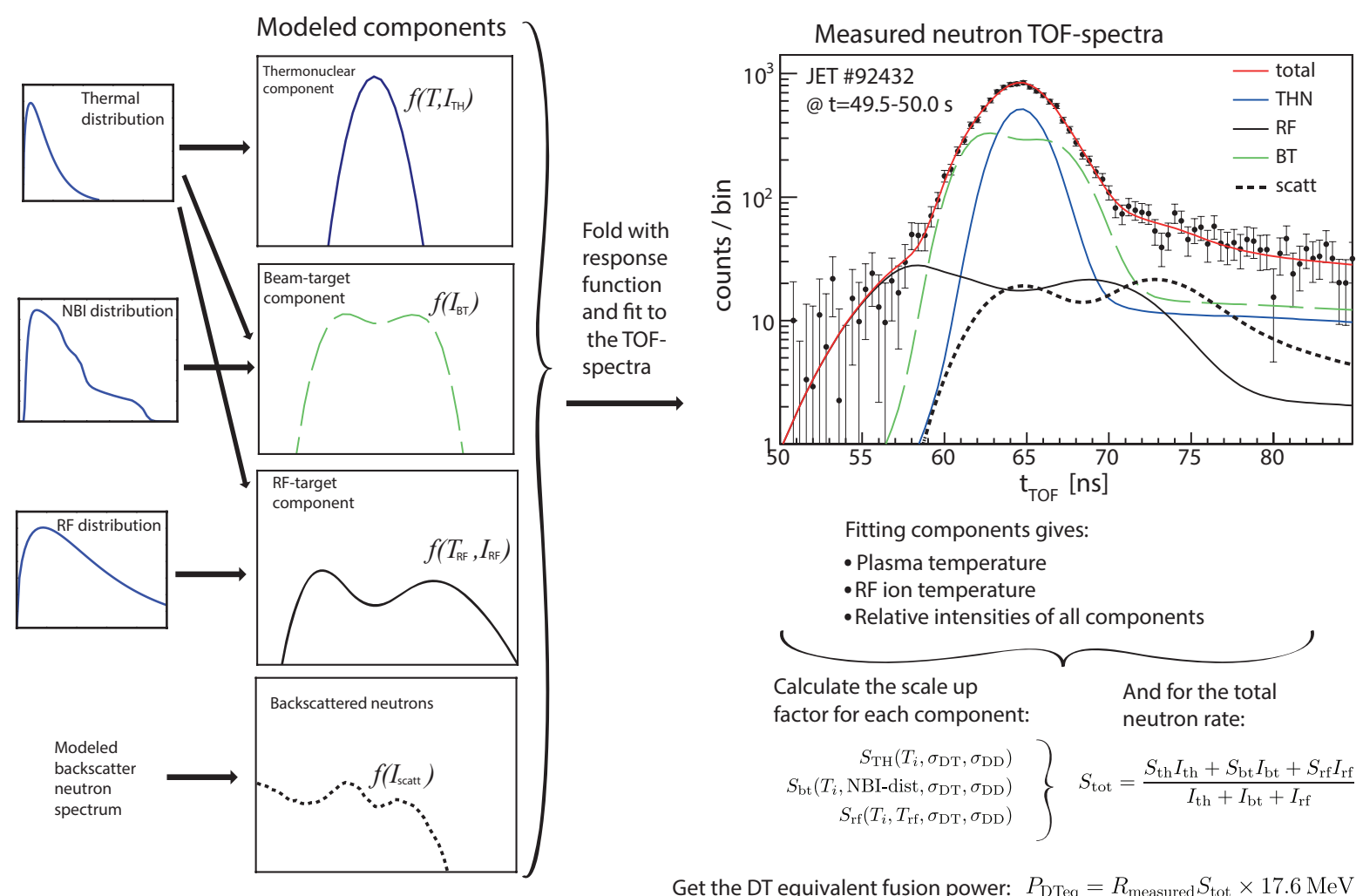

Get the DT equivalent fusion power: $P_{\mathrm{DTeq}}=R_{\text {measured }} S_{\text {tot }} \times 17.6 \mathrm{MeV}$

Fig. 4: An illustration of the process of determining the DT equivalent fusion power from neutron emission spectrometry.

\section{Benchmarking and validation}

\subsection{Comparison with JESTORR and TRANSP based DT predictions}

The DT predictions performed with neutron emission spectrometry have been compared to DT extrapolations conducted using the codes TRANSP[6] and JESTORR[5].

TRANSP is an interpretative transport code often utilized in the field of tokamak-fusion research. Though it takes hours to execute, it can model numerous plasma properties and is here used for simulating the neutron rate.

With TRANSP the DT extrapolations comprise one interpretative simulation of the pulse and one identical run where half of the plasma is tritium. The ratio between the neutron rates from the simulations with DT and DD plasmas correspond to the scale-up factor in equation (14).

JESTORR is a newer physics-model based code, which simulates fusion reaction rates. This code has a built-in function which quickly estimates the DT equivalent fusion power of a JET DD pulse.

Since JESTORR cannot model RF-target reactions and simulating them with TRANSP takes a long time, this component is not used in the comparison.

Three pulses have been used for this comparison with TRANSP and JESTORR. A summary of the pulses' properties is presented in table 4. The TRANSP-simulations of 86614 is a special case since it only has a reliable ion temperature measurement in the time-region 8.0-8.4 s. Therefore, the rest of this TRANSP simulation is omitted from the following comparisons.

Tab. 4: Properties of the three JET pulses used for comparison with JESTORR and TRANSP based DT extrapolations.

\begin{tabular}{ccccccc} 
Pulse & $B_{T} \mathrm{~T}$ & $I_{p} \mathrm{MA}$ & $n_{e} \mathrm{~m}^{-3}$ & $T_{e} \mathrm{keV}$ & $P_{\text {Heat }} \mathrm{MW}$ & $R_{\text {neut }} \mathrm{s}^{-1}$ \\
\hline 92436 & 2.8 & 3.0 & $8 \times 10^{19}$ & 6 & 33.2 & $2.5 \times 10^{16}$ \\
87312 & 3.4 & 2.8 & $8.2 \times 10^{19}$ & 8 & 20.5 & $4.5 \times 10^{15}$ \\
86614 & 2.9 & 2.8 & $7.5 \times 10^{19}$ & 8.2 & 27 & $2 \times 10^{16}$
\end{tabular}


One step in obtaining an accurate estimation of DT equivalent fusion power is a reaction component analysis. Figure 5 shows the comparison between the relative reaction component intensities estimated by NES, compared to the one from JESTORR and TRANSP. For most pulses, TRANSP, JESTORR, and NES make similar component assessments; however, discharge 86614 presented in figure $5 \mathrm{a}$ and $5 \mathrm{~d}$ is an exception. While NES and JESTORR estimate a pulse dominated by beam-target reactions, the TRANSP simulation calculates a more substantial thermonuclear fraction.
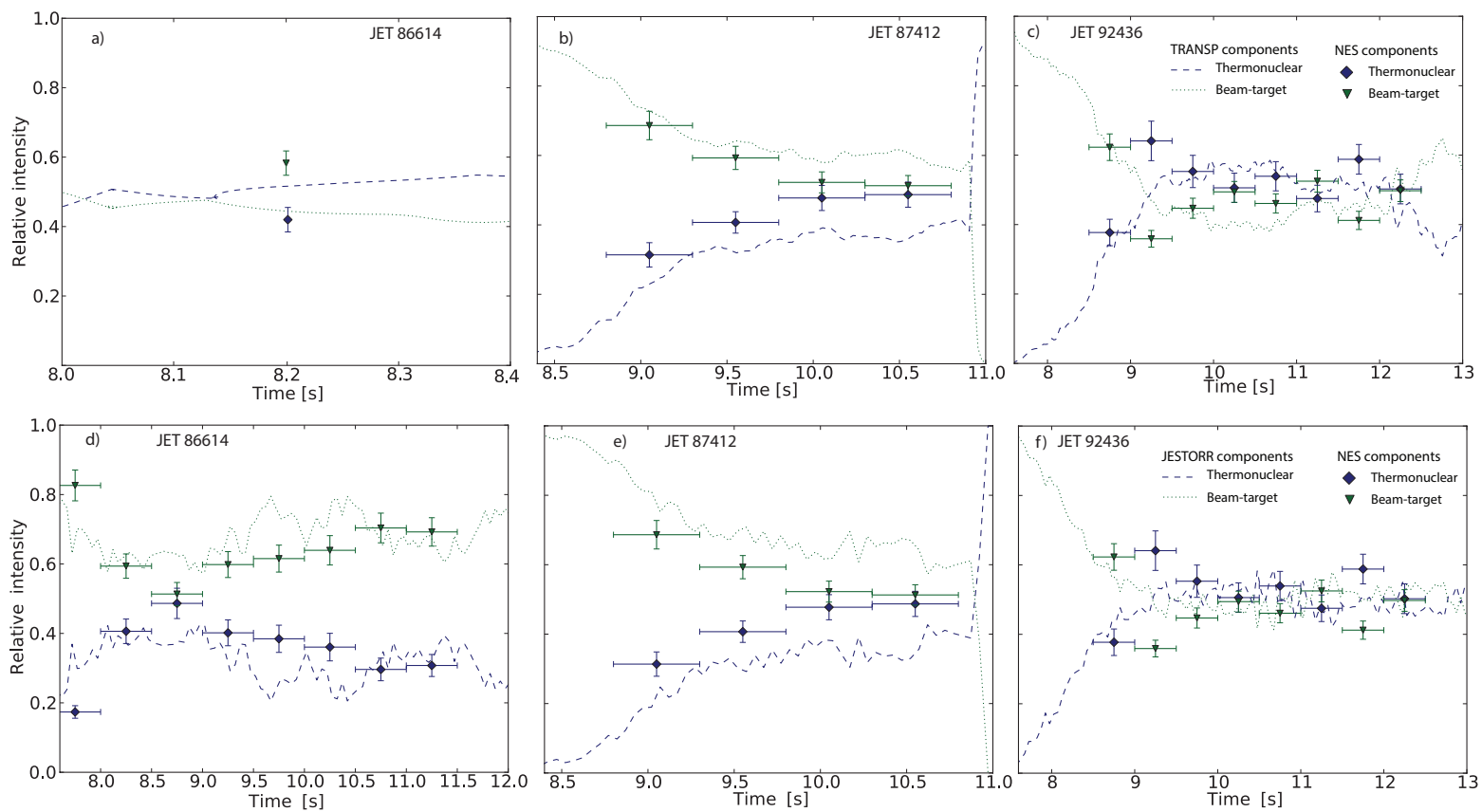

Fig. 5: The relative reaction component intensity for thermonuclear and beam-target reactions estimated NES, compared to those estimated by TRANSP for JET discharge 86614 (a), 87412 (b), and 92436 (c), and compared to those estimated by JESTORR for the same pulses in (d), (e), and (f) respectively.

Once the relative reaction component intensities are determined, we calculate a scale-up factor for each component. The component scale-up factors JESTORR and TRANSP produce are compared to the ones determined by NES, as shown in figure 6 .

Of the two scale-up factors compared, the thermonuclear one is easiest to determine since it only depends on the ion temperature, by the relation shown in figure 1. The beam-target scale-up factor, however, depends both on the bulk ion temperature and from an assessment of the slowing down distribution of the beam ions. For this reason, there is often a more precise agreement when comparing the thermonuclear scale-up factors than the beam-target ones. Consistently, NES estimates the lowest beam-target scale-up factor, while JESTORR estimates the largest one. 

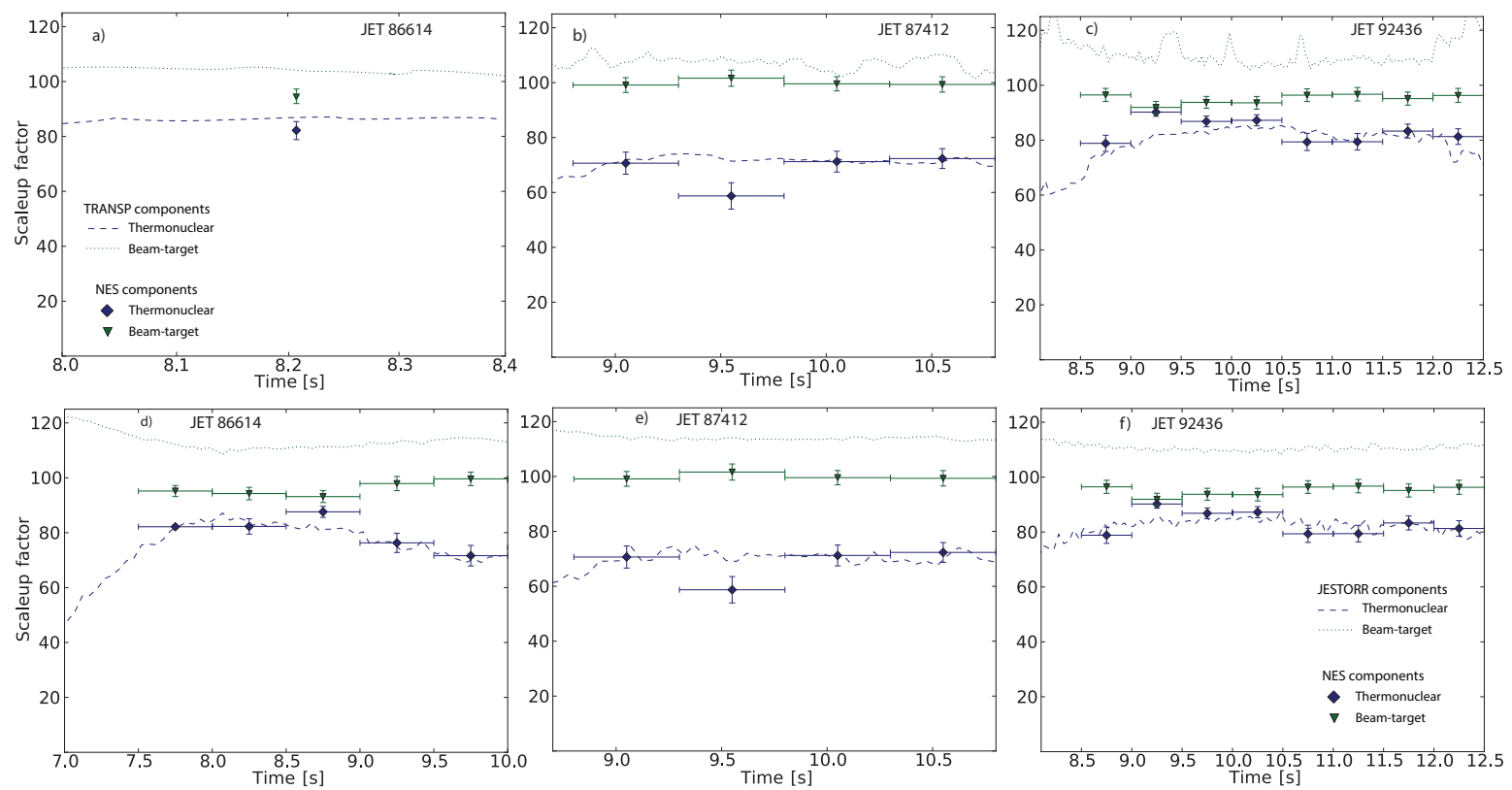

Fig. 6: The component scale-up factors estimated by NES compared to those estimated by TRANSP for JET discharge 86614 (a), 87412 (b), and 92436 (c), and compared to those estimated by JESTORR for the same pulses in $(\mathrm{d}),(\mathrm{e})$, and (f) respectively.

When both the relative component intensities, and the scale-up factors have been established, a total scale-up factor is calculated from equation (14) and we can calculate the DT equivalent neutron rate. A comparison between the DT equivalent neutron rate determined by JESTORR, TRANSP, and NES is displayed in figure 7 . We see a good agreement between the three tested methods, where NES systematically seems to make the most conservative predictions and JESTORR makes the most optimistic one.
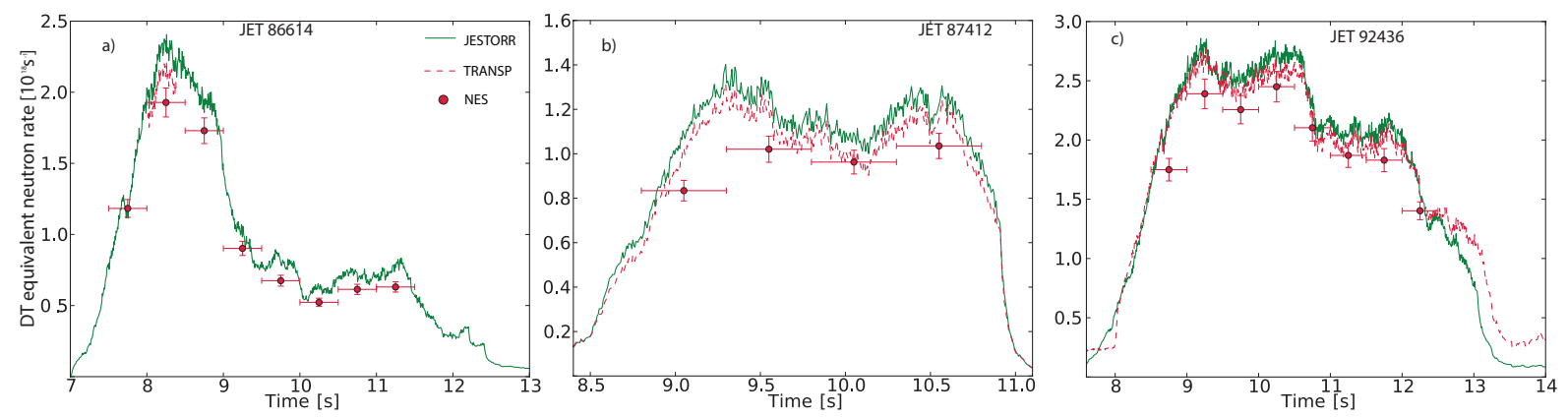

Fig. 7: DT equivalent neutron rate of three JET pulses determined with JESTORR and TRANSP, and NES.

\subsection{Comparison with DTE1 pulses}

As another form of benchmarking, the NES based DT predictions have been compared with pulses from the previous DT campaign at JET, DTE1. Two DTE1 pulses aiming at a fuel-ion ratio of one and having a sustained neutron rate for a few seconds are used in the comparison. For each DT pulse a similar DD shot has been identified and analyzed. The DD and DT pulses used in the comparison are JET shots 77721 and 77946 for DD and 42982 and 42983 for DT. The pulses are presented in table 5 .

The DD pulses have been extrapolated to DT using NES, and their resulting DT equivalent neutron rate has been compared to the measured neutron rate in corresponding DTE1 pulses. Additionally, the neutron spectrometer MPR[17] has been used to analyze the DTE1 shots, to determine its reaction component intensities, which are compared to the extrapolated intensities. 
Tab. 5: The pulses used for comparing DT predictions to similar DTE1 shots.

\begin{tabular}{ccccccc} 
Pulse & Fuel & $n_{e} \mathrm{~m}^{-3}$ & $T_{e} \mathrm{keV}$ & $P_{\mathrm{NBI}} \mathrm{MW}$ & $P_{\text {icrh }} \mathrm{MW}$ & $I_{p} \mathrm{MA}$ \\
\hline 42982 & DT & $8 \times 10^{19}$ & 7 & 22 & 2.0 & 3.8 \\
77721 & DD & $8 \times 10^{19}$ & 6.3 & 21.3 & 2.3 & 3.5 \\
\hline 42983 & DT & $10 \times 10^{19}$ & 6 & 21.7 & 2.2 & 3.4 \\
77946 & DD & $9 \times 10^{19}$ & 7 & 22.8 & 2.7 & 3.45
\end{tabular}

Figure 8 shows the comparison between extrapolated DD pulses and similar DTE1 pulses. In both comparisons the DD pulse has been translated in time to begin in sync with the DT pulse, to facilitate the comparison. We see a good agreement between the DTE1 pulses, and the DT equivalent neutron rate of their analogous deuterium-only shots. The similarities hold for both the total neutron rate and the reaction components, especially in the comparison presented in figure 8a. We must remember, that we do not expect a perfect agreement, since the DD-DT pair corresponds to two different, although similar, pulses.

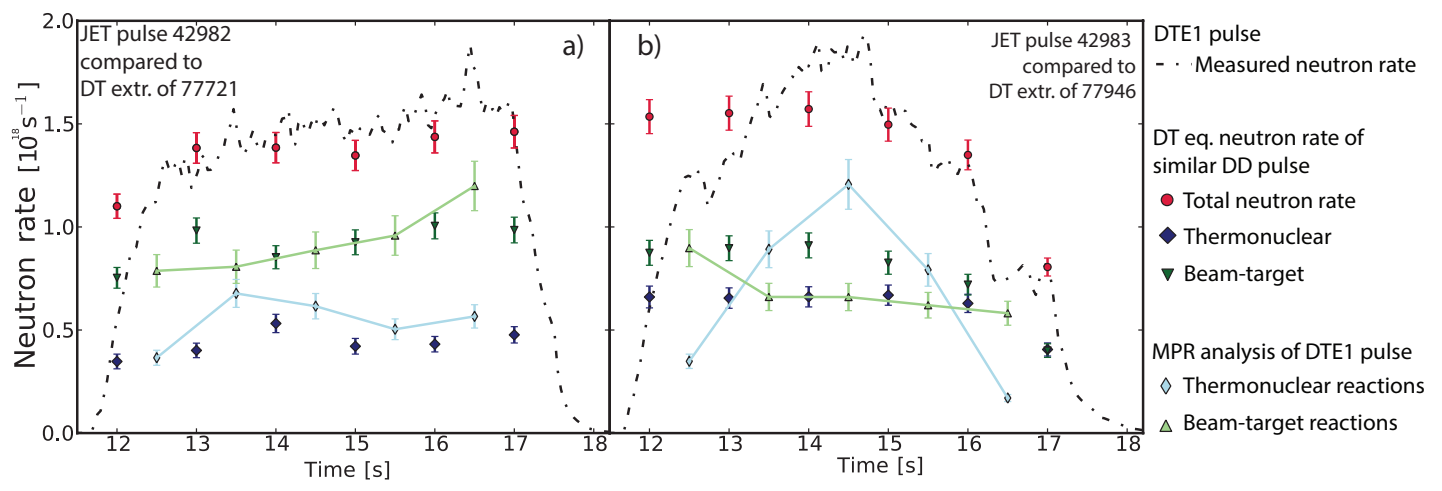

Fig. 8: A comparison between the neutron rate from two DTE1 pulses, and DT extrapolations of similar DD pulses. In (a) shot 42982 is compared to 77721 and in (b) shot 42983 is compared to 77946. The component separations of the DTE1 shots from an MPR analysis is also shown, together with the corresponding extrapolated component intensities.

\section{Results and discussion}

\subsection{Upscaled pulses}

Several JET pulses have been extrapolated to DT with neutron spectrometry and their resulting fusion power is shown in figure 9a. Discharges run in Hybrid[18] and Baseline[19] scenarios are highlighted, as well as those from before the JET Iter-like wall (ILW) upgrade, i.e. with the Carbon wall

The currently best performing D pulses are estimated to reach 6-7 MW if they were run with a DT plasma. If we on the other hand look specifically at the thermal component of the neutron rate, which is the component most interesting for future fusion reactors, its estimated DT fusion power is shown in figure $9 \mathrm{~b}$. 

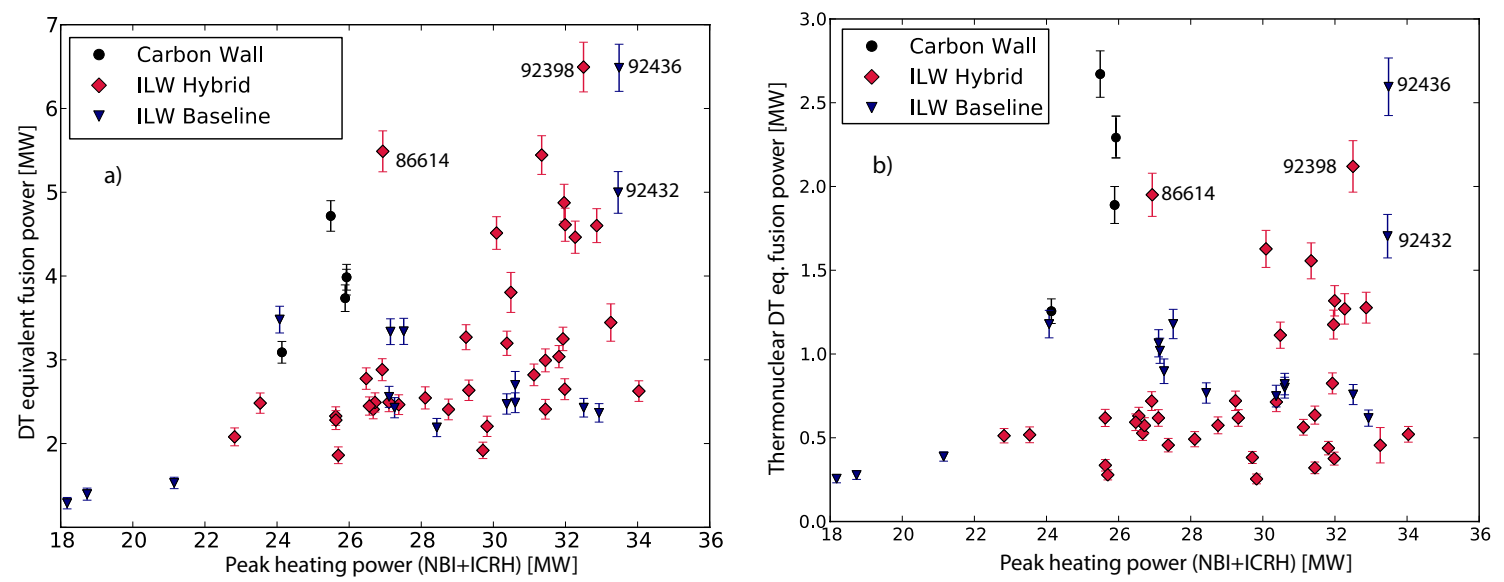

Fig. 9: Estimated DT equivalent fusion power of a set of DD discharges at JET. In (a) the total DT fusion power is presented, and in (b) we see only the thermonuclear contribution to the fusion power.

The older shots from when JET had a carbon wall are seen to be more dominated by the thermonuclear reaction component than those from the new ITER-like wall, possibly because these discharges were run with a higher plasma current, which enabled a higher plasma density at a similar temperature.

\subsection{Prospects for DTE2}

In the forthcoming DT campaign, one aim is to produce $15 \mathrm{MW}$ of continuous fusion power prolonged over a few seconds, with $40 \mathrm{MW}$ input power for heating. Predicted DT equivalent power for a selection of high performance ILW pulses at JET, are displayed in figure 10. A curve, $P_{\text {DTeq }} \propto P_{\text {heat }}^{a}$, has been fitted to the data, and we see that the estimated DT equivalent fusion power scales approximately as $\propto P_{\text {heat }}^{2.3}$, a value in rough agreement with the scaling from [20].

Since the most high-performing JET DD pulses amount to roughly $7 \mathrm{MW}$ of DT equivalent power, we are far off from the desired $15 \mathrm{MW}$. Extrapolating the trend in figure 10 shows that even with 40 MW of heating power, achieving $15 \mathrm{MW}$ will be a challenge. Reaching this level of fusion power is not unreasonable though, given that pulses are scattered around the fitted curve, and the most highperforming pulses lie above this trajectory. Shot 86614, for example, is $30 \%$ over the fitted curve, and a pulse as far above the trend line at the $40 \mathrm{MW}$ mark would just about reach the DTE2 goal of $15 \mathrm{MW}$. 


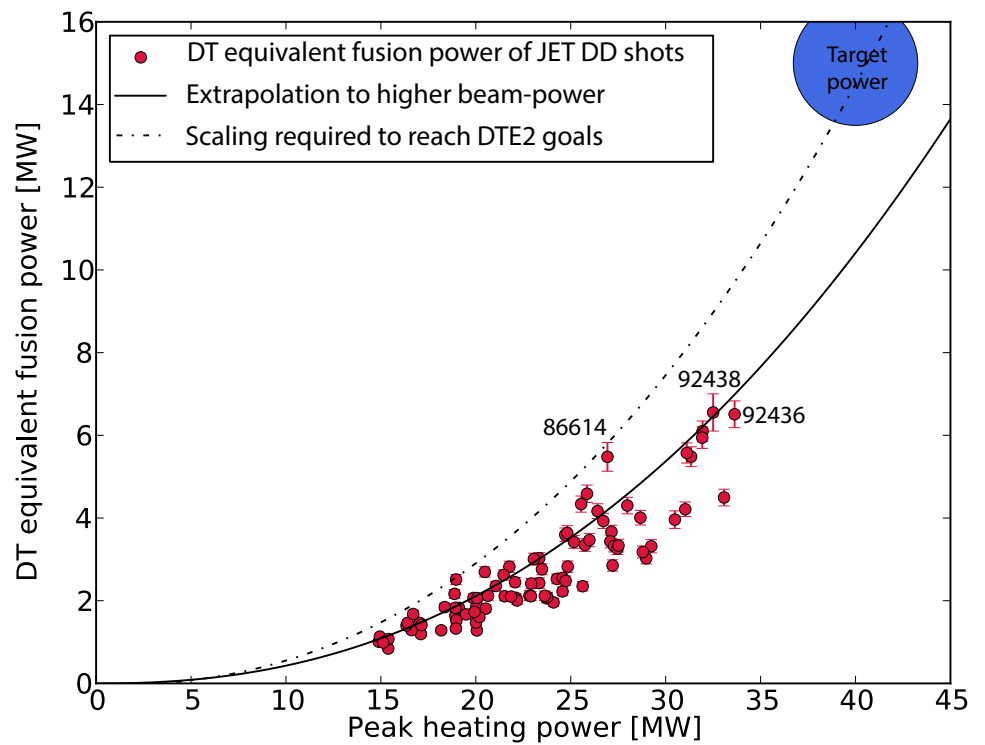

Fig. 10: DT equivalent fusion power of several ILW JET pulses, determined by NES, presented as a function of input heating power. The trend is extrapolated to greater heating power to estimate the possible fusion power in the upcoming DT campaign, where the heating power is expected to be $40 \mathrm{MW}$.

\section{Conclusions}

This paper has presented a method for generating quick and robust estimations of DT equivalent fusion power by analyzing neutron emission spectroscopy (NES) measurements. The developed technique has been compared to DT predictions made with the codes TRANSP and JESTORR, and they are all shown to deviate less than $10 \%$ from each other.

The evaluation of DT equivalent fusion power is sensitive to the assessment of the relative reaction component intensities, i.e., what fraction of the reactions involve the bulk plasma, beam-ions, and RFaccelerated ions, respectively. A strength of DT extrapolation with NES is that it uses measured values of the reaction component intensities, as opposed to modeled ones. Another upside to NES is that it takes the RF-target reaction component into account. Simulating this component in TRANSP takes a long time, and JESTORR has not implemented such a functionality. Both the DT predictions with NES and JESTORR are sufficiently fast to be performed on an inter-shot basis, while TRANSP requires several hours for a simulation.

Based on the benchmarks with other methods, and a comparison with pulses from the previous DT campaign we conclude that NES provides the means for DT extrapolation and that the time-of-flight spectrometer TOFOR grants sufficient information about reaction component intensities and plasma properties to make a robust estimation of DT equivalent fusion power.

In the upcoming DT campaign at JET, DTE2, scheduled next year, the aim is to produce $15 \mathrm{MW}$ of fusion power for five seconds, using $40 \mathrm{MW}$ of input power. The highest performing DD discharges at JET to date are estimated to yield 6-7 MW of fusion power if they were run with a 50/50 DT mixture; however, so far only $33 \mathrm{MW}$ of heating power has been used. Extrapolating the estimated DT equivalent fusion power to an input power of $40 \mathrm{MW}$ shows that the DTE2 goals are achievable but challenging. The forecast shows no problems reaching past $10 \mathrm{MW}$ of fusion power with $40 \mathrm{MW}$ of input power; however, merely the most high-performing discharges can be expected to reach the goal of $15 \mathrm{MW}$.

\section{Acknowledgments}

This work has been carried out within the framework of the EUROfusion Consortium and has received funding from the Euratom research and training programme 2014-2018 and 2019-2020 under grant agreement No 633053. The views and opinions expressed herein do not necessarily reflect those of the European Commission. 


\section{References}

[1] J. Paméla, F. Romanelli, M. L. Watkins, A. Lioure, G. Matthews, V. Philipps, T. Jones, A. Murari, A. Géraud, F. Crisanti, and R. Kamendje, "The JET programme in support of ITER," Fusion Engineering and Design, vol. 82, no. 5-14, pp. 590-602, 2007.

[2] M. Keilhacker and M. L. Watkins, "DT experiments in the JET tokamak," Journal of Nuclear Materials, vol. 266-269, pp. 1-13, 1999.

[3] R. V. Budny, M. G. Bell, H. Biglari, M. Bitter, C. E. Bush, C. Z. Cheng, E. D. Fredrickson, B. Grek, K. W. Hill, H., Hsuan, A. C. Janos, D. L. Jassby, D. W. Johnson, L. C. Johnson, B. LeBlanc, D. C. McCune, D. R. Mikkelsen, H.K., Park, A. T. Ramsey, S. A. Sabbagh, S. D. Scott, J. F. Schivell, J. D. Strachan, B. C. Stratton, E.J., Synakowski, G. Taylor, M. C. Zarnstorff, and S. J. Zweben, "Simulations of deuterium-tritium experiments in TFTR," Nuclear Fusion, vol. 32, no. 3, p. 429, 1992.

[4] M. Gatu Johnson, L. Giacomelli, A. Hjalmarsson, J. Källne, M. Weiszflog, E. Andersson Sundén, S. Conroy, G. Ericsson, C. Hellesen, E. Ronchi, H. Sjöstrand, G. Gorini, M. Tardocchi, A. Combo, N. Cruz, J. Sousa, and S. Popovichev, "The 2.5-MeV neutron time-of-flight spectrometer TOFOR for experiments at JET," Nuclear Instruments and Methods in Physics Research, Section A: Accelerators, Spectrometers, Detectors and Associated Equipment, vol. 591, no. 2, pp. 417-430, 2008.

[5] C. D. Challis. private communication about JESTORR, 2018.

[6] R. J. Goldston, D. C. McCune, H. H. Towner, S. L. Davis, R. J. Hawryluk, and G. L. Schmidt, "New techniques for calculating heat and particle source rates due to neutral beam injection in axisymmetric tokamaks," Journal of Computational Physics, vol. 43, no. 1, pp. 61-78, 1981.

[7] P. H. Rebut and B. E. Keen, "The JET Experiment: Evolution, Present Status, and Prospects," Fusion Technology, vol. 11, no. 1, pp. 13-42, 1987.

[8] L.-G. Eriksson, M. J. Mantsinen, F. G. Rimini, F. Nguyen, C. Gormezano, D. F. H. Start, and A. Gondhalekar, "ICRF heating of JET plasmas with the third harmonic deuterium resonance," Nuclear Fusion, vol. 38, no. 2, p. 265, 1998.

[9] A. Pankin, D. McCune, R. Andre, G. Bateman, and A. Kritz, "The tokamak Monte Carlo fast ion module NUBEAM in the national transport code collaboration library," Computer Physics Communications, vol. 159, no. 3, pp. 157-184, 2004.

[10] T. H. Stix, "Fast-wave heating of a two-component plasma," Nuclear Fusion, vol. 15, no. 5, p. 737, 1975.

[11] H. Brysk, "Fusion neutron energies and spectra," Plasma Physics, vol. 15, no. 7, pp. 611-617, 1973.

[12] M. Gatu Johnson, S. Conroy, M. Cecconello, E. A. Sundén, G. Ericsson, M. Gherendi, C. Hellesen, a. Hjalmarsson, a. Murari, S. Popovichev, E. Ronchi, M. Weiszflog, and V. L. Zoita, "Modelling and TOFOR measurements of scattered neutrons at JET," Plasma Physics and Controlled Fusion, vol. 52, no. 8, p. $085002,2010$.

[13] A. Hjalmarsson, Development and Construction of a 2.5-MeV Neutron Time-of-Flight Spectrometer Optimized for Rate (TOFOR). PhD thesis, Uppsala University, Department of Neutron Research, 2006.

[14] J. Eriksson, S. Conroy, E. Andersson Sundén, and C. Hellesen, "Calculating fusion neutron energy spectra from arbitrary reactant distributions," Computer Physics Communications, vol. 199, pp. 4046, 2016.

[15] L. Ballabio, Calculation and measurement of the neutron emission spectrum due to thermonuclear and higher-order reactions in tokamak plasmas. PhD thesis, Uppsala University, 2003.

[16] W. Cash and W., "Parameter estimation in astronomy through application of the likelihood ratio," The Astrophysical Journal, vol. 228, p. 939, mar 1979.

[17] J. Kllne and H. Enge, "Magnetic proton recoil spectrometer for fusion plasma neutrons," Nuclear Inst. and Methods in Physics Research, A, vol. 311, no. 3, pp. 595-602, 1992. cited By 35. 
[18] C. Gormezano, C. D. Challis, E. Joffrin, X. Litaudon, and A. C. C. Sips, "Chapter 4: Advanced Tokamak Scenario Development at JET," Fusion Science and Technology, vol. 53, no. 4, pp. 958-988, 2008.

[19] T. Casper, Y. Gribov, A. Kavin, V. Lukash, R. Khayrutdinov, H. Fujieda, C. Kessel, and And, "Development of the $\{$ ITER $\}$ baseline inductive scenario," Nuclear Fusion, vol. 54, p. 13005, dec 2013.

[20] M. Keilhacker, "Latest JET results in deuterium and deuterium - tritium plasmas," Plasma Physics and Controlled Fusion, vol. 39, no. 12B, p. B1, 1997. 